\title{
Prácticas y saberes ancestrales de la gastronomía colombiana en el departamento de Cundinamarca, provincia Oriente, $2020^{1}$
}

\section{Colombian gastronomy ancestral practices and knowledges in Cundinamarca department, east province, 2020 \\ (d) https://doi.org/10.52948/sosquua.v3i1.413}

LUIS FELIPE TRIANA CASALLAS

(1) https://orcid.org/0000-0003-4508-4853 Servicio Nacional de Aprendizaje (SENA) (Colombia)

Itrianac@sena.edu.co

SANDRA LILIANA PÉREZ ARTEAGA

(1) https://orcid.org/0000-0002-4280-5873

Servicio Nacional de Aprendizaje (SENA) (Colombia)

sliliaperez@misena.edu.co

Artículo de investigación

Recepción: 18 de junio de 2021

Aceptación: 7 de septiembre de

Cómo citar este articulo:

Triana Casallas, L., y Pérez Arteaga, S. (2021). Prácticas y saberes ancestrales de la gastronomía colombiana en el departamento de Cundinamarca, provincia Oriente, 2020. Sosquua, 3(1), pp. 20-37 https://doi.org/10.52948/

\footnotetext{
1 Este artículo es parte de una investigación asociada al proyecto "Rescate de la memoria de las recetas tradicionales y/o ancestrales, su aplicación en la nueva gastronomía, la seguridad alimentaria y el fortalecimiento del turismo experiencial en el departamento de Cundinamarca" (2019). En este trabajo se realizó la colaboración de Sandra Liliana Pérez Arteaga, autor e instructor investigador en el Centro Nacional de Hotelería, Turismo y Alimentos (CNHTyA) del SENA. Investigación desarrollada y financiada con el auspicio del Sistema de Investigación, Desarrollo Tecnológico e Innovación (SENA), Santa Fe de Bogotá, Colombia.
} 


\section{Resumen:}

Con la investigación se recopiló información sobre prácticas y saberes de la gastronomía colombiana, provincia Oriente en Cundinamarca, buscando rescatarcostumbresalimentariasalinteriordelasfamilias, llevandounregistro escrito de ellas para mostrarlas como identidad cultural. La metodología utilizada es cualitativa de tipo exploratoria, aplicada desde la perspectiva de Fals Borda, Investigación Acción, con la participación de portadoras de tradición y de campesinos en acciones participativas (Mingas). El diseño fue fenomenológico hermenéutico, que se concentra en la interpretación de la experiencia humana y los "textos" de la vida (Ayala Carabajo, 2008). El estudio consistió en la búsqueda de semillas nativas y endémicas casi extintas, para luego su cultivo en huertas caseras, tipo piloto, con prácticas tradicionales planificadas. Se obtuvo como resultado la recuperación de 11 productos agrícolas: sagú, arrayanes, malanga, malangay, coyo blanco con morado, coyo amarillo con morado, manguzán, caimitos, nísperos, frijol baluy y bejuco batata. A la par de la siembra, se identifican personas locales portadores de conocimiento culinario generacional, se recopilan 40 recetas culinarias preparadas a base de productos agrícolas rescatados y bajo métodos y técnicas tradicionales de preparación y cocción. Para dar a conocer los productos culinarios y promocionar la región, se crearon tres circuitos turísticos motivando a la salvaguardia de los saberes locales.

Palabras clave: saberes; gastronomía; ancestro; recetas; convites; legado; stakeholders.

\section{Abstract:}

With the research, information was collected on practices and knowledge of Colombian gastronomy, Oriente province in Cundinamarca, seeking to rescue food customs within families, keeping a written record of them to show them as cultural identity. The methodology used is qualitative of an exploratory type, applied from the perspective of Fals Borda, Research Action, with the participation of bearers of tradition and peasants in participatory actions (Mingas). The design was hermeneutical phenomenological, that focus on the interpretation of human experience and the "texts" of life (Ayala Carabajo, 2008). The study consisted of the search for native and endemic seeds that were almost extinct, and then their cultivation in pilot-type home gardens, with planned traditional practices. The result was the recovery of eleven agricultural products: sago, myrtles, malanga, malangay, white with purple coyo, yellow with purple coyo, manguzán, caimitos, medlars, baluy beans and sweet potato bejuco. Along with the sowing, local people with generational culinary knowledge are identified, 40 culinary recipes prepared 
based on rescued agricultural products and under traditional methods and techniques of preparation and cooking are collected. To publicize the culinary products and promote the region, three tourist circuits were created motivating the safeguarding of local knowledge.

Keywords: knowledges; gastronomy; ancestor; recipes; banquet; legacy; stakeholders.

\section{Introducción}

En las regiones colombianas existen familias con conocimientos relacionados con la tierra y sus derivados que aportan a la generación de conocimiento en diversas áreas. En especial, cuando se trata de la cocina ancestral y tradicional se siente la necesidad de realizar un rescate de estas prácticas y saberes de la gastronomía, reposados al interior de estos núcleos y que, por razones no claras, se encuentran ocultas o no son mostradas al mundo.

La provincia de Oriente del departamento de Cundinamarca, Colombia, cuenta con una ubicación estratégica por estar a 40 minutos de la capital del país y por ser parte del corredor vial que lleva a la entrada de los llanos orientales. Gracias a la diversidad de sus climas aporta una gran riqueza natural, cultural y agrícola que ha permitido la incubación de talentos reconocidos en la gastronomía autóctona como doña Betty Rojas de Caquezáy el señor Rodríguez de Chipaque, sin dejar de lado otras personalidades menos famosas. A estas personas se les denomina portadores de tradición oral de conocimientos, en su mayoría son mujeres de origen campesino que por muchas circunstancias de la vida no han tenido el privilegio de ir a una academia a recibir conocimientos de formación técnica-profesional. Sin embargo, con su sabiduría en la praxis son ellas quienes transmiten los conocimientos, en algunos casos por herencia familiar como "expertos empíricos".

Cundinamarca no cuenta con una documentación relacionada con especificaciones de la tradición culinaria. Por tal razón, se evidencia que la cultura gastronómica del departamento sea conocida solo superficialmente, predominando información sobre la cocina santafereña como punto central de una extensa región, olvidando la existencia de los 116 municipios que conforman el departamento y la riqueza culinaria que posee. Lo anterior, sin desconocer el fenómeno de desplazamiento que desde siempre ha existido y que deriva en la migración y el establecimiento de muchas recetas en diferentes zonas geográficas, aportando al enriquecimiento cultural gastronómico (Acosta López, 2018).

En la provincia de Oriente aún existe la posibilidad de rescatar una gran variedad de productos, semillas y frutas; tristemente se encuentran en vía de extinción por el fenómeno de la poca demanday la necesidad de los campesinos de producir cosechas más rápidas. Por consiguiente, uno de los objetivos del 
proyecto de investigación ha sido recuperar las semillas que aún se pueden salvar y caracterizar, para luego ser donadas a los agricultores que apoyen en la producción, el inventariado y la creación de huertas caseras. El fin es obtener el producto y multiplicarlo entre los pobladores de la provincia que se quieran unir de forma voluntaria a este proceso de conservación. Al multiplicar esta acción se espera poder recuperar la culinaria ancestral predominante en la zona.

La importancia de preservar la cocina tradicional como patrimonio de la humanidad se refleja en la necesidad de proteger junto con ella todos esos instrumentos que permiten a cada plato existir. Por tal razón, ha adquirido un reconocimiento a nivel internacional como se detalla a continuación:

Las culturas alimentarias constituyen de ahora en adelante un nuevo paradigma patrimonial, partícipe de la "efervescencia" (Quintero, 2005) introducida por la Convención para la salvaguardia del patrimonio cultural inmaterial (Unesco, 2003), debido a su relación estrecha con las nociones de cultura - en su sentido antropológico - de memoria y de identidad. (Matta, 2011, p. 2)

Ante este hecho, se ratifica la transmisión de saberes de forma generacional como parte del patrimonio cultural de los pueblos como muestra de identidad, arraigo y respeto por las tradiciones culinarias propias de la región. Según Meléndez y Cañez De la Fuente (2009), la práctica del rescate de la cocina ancestral permite generar una propuesta en la nueva cocina haciendo uso de técnicas modernas en la elaboración e implementación de tecnología. Esta afirmación contribuye para Colombia en la sostenida mejora de la seguridad alimentaria de las comunidades en las provincias de Cundinamarca, desencadenando un fortalecimiento a la cadena de valor asociada al turismo.

En relación con lo expuesto, la presente investigación se enfoca en la recuperación de recetas, utensiliosy el legado de las costumbres alimentarias de la provincia "Oriente" de Cundinamarca como fundamental, realizando grandes aportes a la cocina colombiana como es el ligar la producción agrícola y de autoconsumo local a un modo de vida autosuficiente y accesible alimentariamente durante todo el año. De esta manera, permitiendo a las mujeres en el seno de la unidad doméstica utilizar estos productos agrícolas en la preparación de recetas familiares obtenidas de forma generacional como reflejo de identidad cultural. Por esta razón, se busca continuar y ampliar el proceso de recuperación de las tradiciones culinarias y de redescubrir todo lo que se encuentra oculto en el interior de las familias del departamento de Cundinamarca.

Para la realización de esta investigación se tuvo el apoyo técnico y financiero del Servicio Nacional de Aprendizaje (SENA), por medio del Centro Nacional de Hotelería, Turismo y Alimentos (CNHTyA), a través del Sistema de Investigación Desarrollo Tecnológico e Innovación (SENNOVA) y de sus estrategias para el fortalecimiento en los estándares de calidad y pertinencia 
mediante la investigación aplicada. Lo anterior, dando cumplimiento a la búsqueda y gestión de conocimiento, gracias a la colaboración de las comunidades pertenecientes a la región de la provincia de "Oriente", en Cundinamarca; aprendices semillero de investigación e instructores investigadores.

Este artículo hace parte del producto de investigación aplicada para la gestión de conocimiento SENA - CNHTyA (2019).

\section{Objetivo general.}

Rescatar las costumbres culturales ancestrales gastronómicas y corredores turísticos de la región Oriente de Cundinamarca.

\section{Objetivos específicos.}

- Determinar el impacto del sector productivo, en la creación de nuevos circuitos turísticos en armonía con las costumbres culturales ancestrales gastronómicas y corredores turísticos de la región oriente de Cundinamarca.

- Determinar el impacto ambiental en la siembra de semillas de productos agrícolas endémicos y nativos dentro de las costumbres culturales ancestrales gastronómicas y corredores turísticos de la región oriente de Cundinamarca.

- Determinar el impacto sociocultural, desde el empoderamiento de la comunidad en las costumbres culturales ancestrales gastronómicas y corredores turísticos de la región Oriente de Cundinamarca.

- Determinar el impacto tecnológico de nuevas herramientas, para la elaboración de platos típicos desde las costumbres culturales ancestrales gastronómicas y corredores turísticos de la región oriente de Cundinamarca.

- Determinar el impacto de la participación de los aprendices del centro de formación, en la investigación aplicada bajo la praxis, desde las costumbres culturales ancestrales gastronómicas y corredores turísticos de la región oriente de Cundinamarca.

\section{Metodología}

\section{Tipo de investigación.}

La investigación inicia con un corte cualitativo, se realizó desde la perspectiva latinoamericana Investigación Acción Participativa (IAP) de Orlando Fals Borda, quien sugiere: "es necesario descubrir una base para entender los vínculos que existen entre el desarrollo del pensamiento científico, el contexto cultural y la estructura de poder de la sociedad" (Ortiz y Borjas, 2008). 
La característica predominante de la IAP es la forma de producir el conocimiento a través de la colectividad. Asimismo, el vivir experiencias recurriendo al diálogo de forma participativa y voluntaria, transformando a cada uno de los actores desde su propia realidad, al aporte colectivo de la sociedad.

\section{Diseño de la investigación.}

El diseño fue fenomenológico hermenéutico, el cual se basa en describir las experiencias vividas por las personas como parte del dominio de la práctica culinaria, basado en:

Se concentra en la interpretación de la experiencia humana y los "textos" de la vida. No sigue reglas específicas, pero considera que es producto de la interacción dinámica entre las siguientes actividades de indagación: a) definir un fenómeno o problema de investigación (una preocupación constante para el investigador), b) estudiarlo y reflexionar sobre éste, c) descubrir categorías y temas esenciales del fenómeno (lo que constituye la naturaleza de la experiencia), d) describirlo y e) interpretarlo (mediando diferentes significados aportados por los participantes). (Hernández Sampieri, Fernández y Baptista, 2014, p. 494)

El proyecto se realizó con los siguientes pasos dentro de la investigación:

Figura 1. Pasos de la investigación

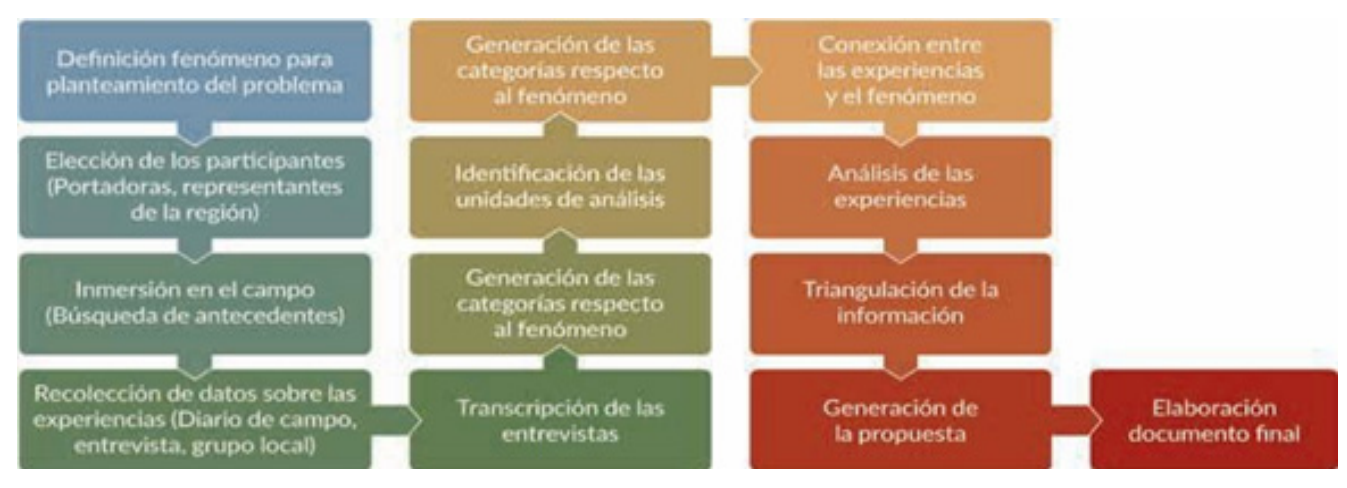

Fuente: Pérez y Carrillo, 2020.

\section{Población.}

El departamento de Cundinamarca está conformado administrativamente por 15 provincias que agrupan un total de 116 municipios. Estas provincias se analizaron con el objetivo de seleccionar una que presentara alto impacto, razón por la cual la provincia de Oriente resaltó al encontrarse 
en el camino hacia Villavicencio y por ser de obligatorio recorrido hacia los Llanos Orientales del país. Se seleccionó como muestra las comunidades de los municipios de Cáqueza, Une, Chipaque, Fosca, Gutiérrez, Guayabetal, Ubaque, Choachí, Fómeque y Quetame; en específico, representantes de agricultores, portadoras de tradición y custodios de semillas, los cuales con sus conocimientos, experiencias y aportes, facilitaron el desarrollo de la investigación.

\section{Muestra.}

Los criterios para seleccionar la muestra corresponden al orden cualitativo, haciendo elección de los elementos sin dependerse de la probabilidad, también conocido como muestras no probabilísticas. Es decir, el procedimiento corresponde al criterio y a la toma de decisiones otorgada por el grupo de investigadores en relación con las características dadas por la investigación, como es el caso particular de pobladores que accedieron de manera voluntaria a participar, basado en un estudio exploratorio y descriptivo, para concluir en una perspectiva teórica (Hernández Sampieri, Fernández y Baptista, 2014). Las muestras no probabilísticas también se reconocen como muestras dirigidas, ratificando que la elección de casos depende del criterio específico del investigador. A su vez, clarifica que no todos los miembros de la población tienen igualdad de oportunidad para conformarla (Angélica, 2016).

Con base en lo anterior, se parte de una inmersión inicial tomando la similitud cultural, gastronómica y agrícola de la provincia de Oriente, para luego delimitar el grupo en representantes, de acuerdo con las temáticas objeto de estudio, encontrando una muestra final significativa.

El primer grupo seleccionando corresponde a las portadoras de tradición, delimitado por pertenecer a familias antiguas y longevas en la región y cuyos saberes datan de 200 años atrás por tradición oral. También, por ser reconocidas a nivel culinario tradicional y ancestral; al manejar platos representativos de la zona a base de productos agrícolas endémicos y típicos, y por ser las herederas de los saberes de sus antepasados; se toma un representativo de 5 personas: Berta Hernández de Hernández, Carmenza Parrada, Nora Imelda Clavijo, Marcela Duque y Francelina Zambrano.

El segundo grupo corresponde a los agricultores activos en la región, seleccionados por su interés voluntario y deseo en participar, los cuales ofrecieron en sus fincas un espacio para una huerta y desarrollar el proceso de cultivo del estudio. Estos lugares debían poseer unas características especiales marcadas por el tipo de suelo, clima y disposición de agua natural, necesarios para llevar a cabo el proceso de siembra, cuidado y recolección de la producción. La muestra representativa corresponde a cuatro fincas: Finca Sión en la vereda Abasticos, de la señora Mayra Santos; la Granja la María en la vereda Mercadillo I, del señor Luis Carlos Céspedes, la Finca La 
Corraleja en la vereda Tausuta I, del señor José Ramiro Gutiérrez y la Finca la Primavera en la vereda Pantano de Carlo de la señora Olga Ortiz. Al grupo de agricultores se les hizo entrega de semillas de sagú, malanga, chonque, coyo amarillo con morado, coyo blanco con morado, manguzán, frijol baluy, ahuyamilla y bejuco batato, al igual, insumos agrícolas orgánicos para el control de plagas, prevención y tratamiento.

Al ser una muestra no probabilística: "si la población es menor a 50 individuos, la población es igual a la muestra", sustentando, la no necesidad de aplicar un criterio muestral (Angélica, 2016).

\section{Método.}

El método utilizado es la IAP, estimulando desde la realidad la participación activa de los grupos implicados dentro de su entorno. Además, mostrando el contexto sociocultural en el cual viven de forma crítica e invitando a reflexionar la transformación de forma colectiva, desde la praxis. Para alcanzar el acercamiento con el objeto de estudio, se realizaron reuniones de trabajo en equipo de tipo solidario, cooperativo, colaborativo y camaradería alrededor de una tradición campesina como es la comida conocida como el convite o Minga. Un escenario propicio para la recopilación de datos específicos relacionados con la culinaria, sus recetas y métodos de preparación. Por otra parte, a su vez se remembró la historia, su significado e importancia que marca la identidad cultural local, hechos registrados en los diarios de campo, listas de chequeo y formatos de verificación de producto.

La observación como método de indagación significó un papel importante tanto en el proceso culinario, como en el agrícola, pero especialmente en el último. Este permitió corroborar las prácticas agrícolas tradicionales y rudimentarias de tipo generacional ligados a la luna, tiempos de siembra y mano del campesino, tema contundente para recibir una apropiada cosecha.

Para respaldar la información se hizo necesario realizar entrevistas estructuradas y no estructuradas con ambos grupos de muestra, soportados en videos de grabación. Este material permitió clarificar ideas al momento de hacer uso de la investigación descriptiva puntualizando las características de la población que se estaba estudiando y generando un análisis a profundidad. Dando continuidad al proceso de investigación, fue vital la participación voluntaria de la comunidad para la creación de 3 circuitos turísticos. El tema motivacional de viaje es la puesta en escena de la culinaria ancestral y tradicional a base de productos agrícolas endémicos locales, siendo representativos de la identidad cultural local, en conjunto con la expresión natural dada por lugares magníficos a visitar.

La información recopilada fue analizada de forma simultánea comprendiendo los comportamientos subyacentes en la investigación 
cualitativa, basados en la observación, en el lenguaje verbal, no verbal. Además, proporcionando resultados como muestra significativa del comportamiento individual y colectivo alrededor de los saberes locales representativos. Para lograrlo se utilizó un procedimiento interpretativo estándar de preguntas abiertas y de análisis de contenido, con el ánimo de reducir datos, seleccionar palabras claves, agrupar frases en dimensiones y codificar categorías. Esta información se ve reflejada en códigos numéricos transformando los datos cualitativos en datos cuantitativos, manifestándose de manera categorizada, disponible para codificar y tabular, reduciendo la información a datos numéricos (Rodríguez, 2003).

La información obtenida es analizada haciendo uso de la estadística descriptiva, indicando el número de veces que se repite un valor entre la población de la muestra, en concordancia con el objeto de estudio. Dicho hecho facilita el poder mostrar resultados de varias formas, describiendo de forma aproximada el fenómeno de estudio (Monje Álvarez, 2011, p. 174).

\section{Resultados}

La investigación realizada en la provincia de Oriente, en Cundinamarca, permitió generar impactos de alto significado y relevancia para la comunidad involucrada en su desarrollo; reflejadas en los sectores productivo, ambiental, sociocultural, tecnológico, incluso en el centro de formación (SENA). Dentro del proceso de medición y seguimiento se hizo necesario utilizar la matriz de marco lógico aportando a lograr los objetivos planteados a partir de lo técnico-funcional. Esta herramienta facilitó el proceso de investigación desde lo constructivo para los investigadores y el semillero de investigación. De esta manera, consolidando un entendimiento desde lo cualitativo a lo cuantitativo para dar opción a la valoración socioeconómica global (impacto/ propósito) que incluye efectos positivos y negativos; también conocidos como deseados, previstos, no deseados y no previstos. A continuación, se describen los impactos.

\section{Impacto en el sector productivo.}

Figura 2. Platos ancestrales rescatados y circuitos turísticos
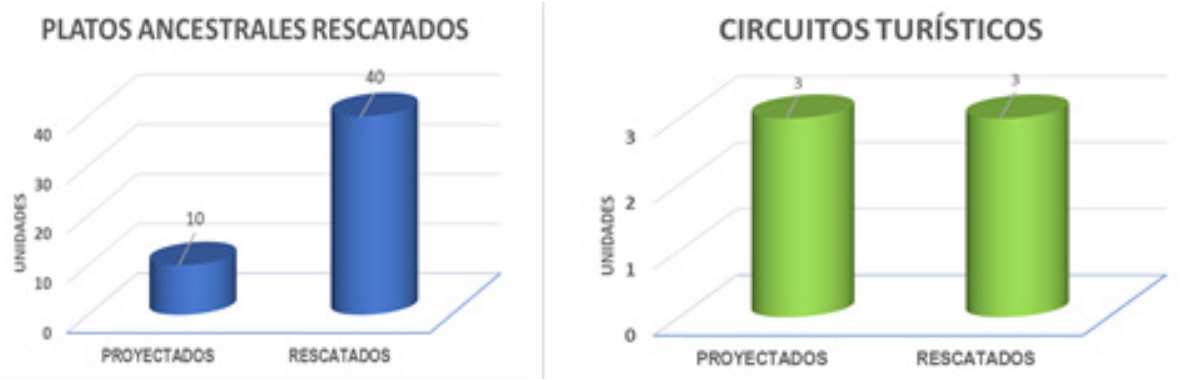

Fuente: Pérez y Carrillo, 2020. 
La búsqueda de semillas endémicas y ancestrales a lo largo de la provincia de Oriente motivó e incentivó la participación de la comunidad al rescate de productos agrícolas representativos de la región y sus métodos tradicionales de siembra y cultivo. De igual manera, reconociendo la existencia del producto, sus cualidades y usos, ocasionó un interés desde lo comercial por adquirir los productos en los lugares de venta de alimentos. Con los convites o Mingas realizadas, logrando la participaron de forma activa y voluntaria una parte significativa y representativa de la comunidad, se identificaron platos culinarios ancestrales y tradicionales con base en los productos agrícolas endémicos y típicos. Este hecho ha incentivado en los pequeños restaurantes a preparar platos sencillos y modestos para manejar de forma progresiva la inclusión del producto preparado para las nuevas y viejas generaciones. Este comportamiento favorable contribuye a fomentar la seguridad alimentaria de la población local en todos los periodos del año.

Se destaca la oportunidad de generar valor a la región desde lo económico, con la propuesta de tres circuitos turísticos. El primero es vía La Calera hacia Cáqueza; el segundo vía Villavicencio a Fosca y el tercero corresponde a la vía Chipaque - Ubaque- Choachí. Son resultado de la participación activa e involucramiento por parte de los aprendices semilleros de investigación del centro SENA CNHTyA, la comunidad e instructores SENA. Para alcanzar este logro, se hizo necesario un reconocimiento por la zona, identificando la gran riqueza natural existente prodigiosamente en los diferentes caminos reales de época ancestrales.

El objeto de los circuitos consiste en recorrer su impacto cultural y los puntos neurales reconocidos como restaurantes típicos en las costumbres locales, que están dispuestos a ofrecer de manera estandarizada los platos gastronómicos de tradición ancestral, conformando un motivo de viaje para turistas nacionales e internacionales. Estos se encuentran disponibles para la gestión pertinente de las autoridades locales con el ánimo de incluirlos en el inventario turístico, promoción y comercialización de estos.

\section{Impacto ambiental.}

Figura 3. Rescate de semillas extintas y huertas caseras
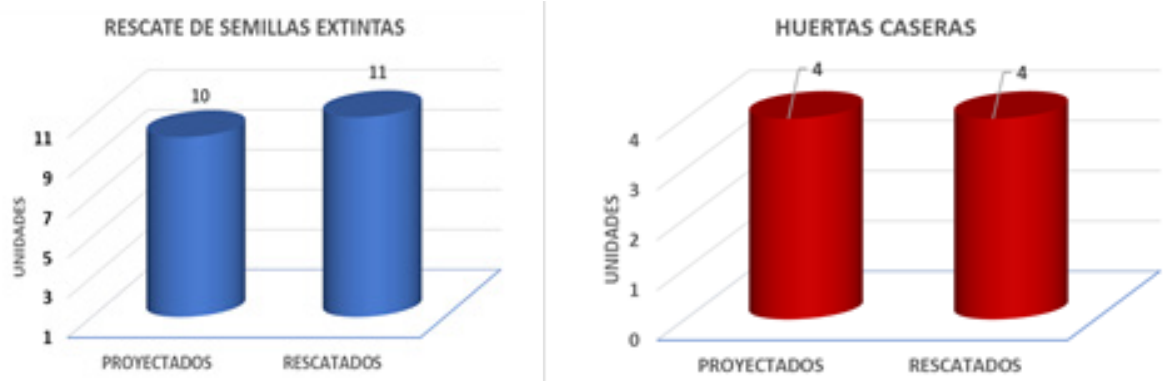

Fuente: Pérez y Carrillo, 2020. 
La invitación realizada a la comunidad participe del proyecto a través de coloquios, convites o Mingas de praxis en el territorio, permitió incentivar a los propietarios de las huertas caceras a la siembra de semillas endémicas y ancestrales de productos agrícolas locales; motivando y propiciando el empoderamiento por cultivar los diferentes productos agrícolas endémicos rescatados; además, aplicando técnicas, métodos y técnicas tradicionales con algunos manejos de herramientas modernas, garantizando un mejor tratamiento del suelo.

El ejercicio realizado con las huertas caseras es un ejemplo de las buenas prácticas agrícolas que favorecen cosechar productos orgánicos en los tiempos prudentes, marcados por la tipología del producto agrícola de origen ancestral del entorno regional de su cultivo. Otra contribución ambiental es la reforestación dada por los árboles de fruto que tienen un proceso de crecimiento prolongado en pro del mejorando de la calidad en el aire y mitigando el proceso de calentamiento global.

\section{Impacto sociocultural.}

Figura 4. Participación de la comunidad en actividades del proyecto

PARTICIPACIÓN DE LA COMUNIDAD EN ACTIVIDADES DEL PROYECTO

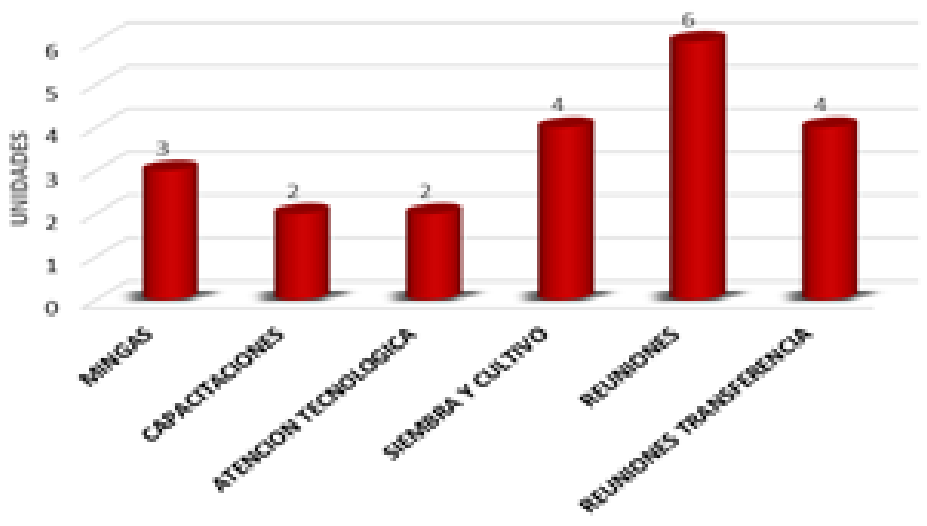

Fuente: Pérez y Carrillo, 2020.

La aplicación del método IAP por medio de trabajo en equipos a través de coloquios, convites o Mingas, propició en los representantes de la comunidad involucrarlos directamente en el proceso investigativo; así como remembrar acontecimientos culturales, hábitos, costumbres importantes locales alrededor de la comida ancestral y tradicional. Movidos por el interés de recordar y apropiar conocimiento sobre la cultura ancestral de 
la región, cada uno de los participantes compartió sus saberes mediante el dialogo y la práctica de actividades diarias con diferentes miembros de las comunidades en los 10 municipios que conforman la provincia.

Las tareas mencionadas anteriormente facilitaron ubicar a los portadores de tradición principales de la región y a las familias que trasmiten empíricamente de generación en generación esos saberes orales. Los encuentros se convirtieron en espacios importantes de interacción periódica tradicional, suministrando de esta manera la recopilación de 40 recetas derivadas del uso de los productos agrícolas rescatados. Así también, aprovechando esta oportunidad para concretar el estudio histórico alrededor de cada preparación estelar, como hecho relevante para la salvaguarda de estos saberes como identidad cultural local.

Un hecho relevante de la investigación se centró en capacitaciones a través de los espacios tradicionales antes mencionados. Asimismo, se dio a conocer el proceso del proyecto y sus avances periódicos a la comunidad en general, resaltando como hecho significativo la instrucción y reaprendizaje en el manejo de la tierra para acondicionarla de forma más adecuada para la siembra; además del respectivo proceso de cosecha, evitando y minimizando el maltratar el suelo y por ende el mejoramiento la cosecha de los productos.

De igual manera, se generaron espacios de transmisión de información y de gestión del conocimiento, específicamente sobre la culinaria representativa y la importancia de ella como acervo cultural para trasferir a las generaciones futuras. Todo lo anterior no hubiese sido posible sin la decidida actitud altruista y responsable, demostrando un alto compromiso de todos actores de la investigación, quienes también participaron en el evento de difusión de Sennova, SENA CNHTyA (2019), en la divulgación y la gestión del conocimiento. Entre otros resultados se encuentra:

\section{Impacto tecnológico.}

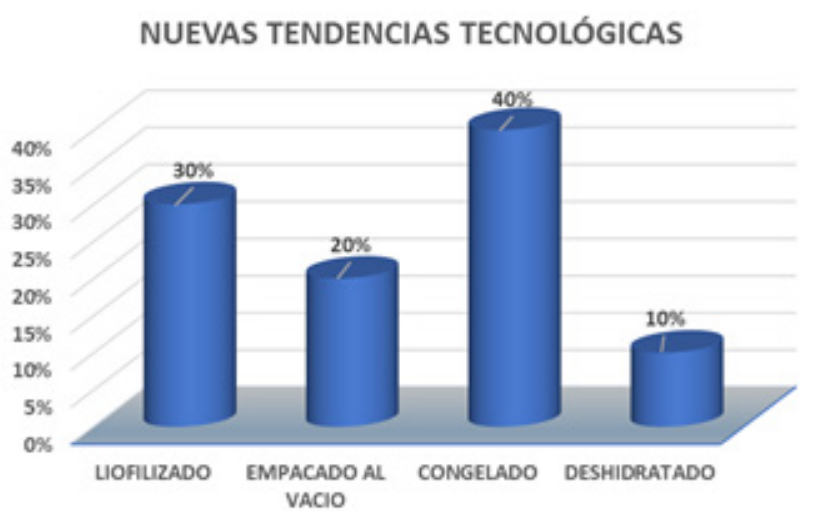

Figura 5. Nuevas

tendencias

tecnológicas

Fuente: Pérez y

Carrillo, 2020. 
Seaplicaron técnicas de manipulación y conservación de los productos con el ánimo de preservarlos por un tiempo máximo de 20 años como materia prima e insumo para la construcción de platos ancestrales y tradicionales, garantizando el patrimonio inmaterial para generaciones venideras. Con la realización de talleres en el laboratorio de Sennova SENA CNHTyA, en los cuales participó el grupo de aprendices semilleros de investigación, las portadoras de tradición e instructoras investigadores; se logró conocer y apropiar conocimientos de liofilización y conservación de materia prima con instructores del área de alimentos del centro SENA. También se trabajó la técnica de emplatado de la comida, con la participación y asesoría del chef Jancarlo Gamonal Cáceres de la República del Perú.

Lo anterior es expresado en el uso de herramientas tecnológicas para la intervención en la presentación de los platos culinarios a partir de las nuevas tendencias gastronómicas, acordes a la demanda emergente.

\section{Impacto de la participación de los aprendices del centro de formación.}

Figura 6. Semillero de investigación y transferencia de conocimiento a la comunidad
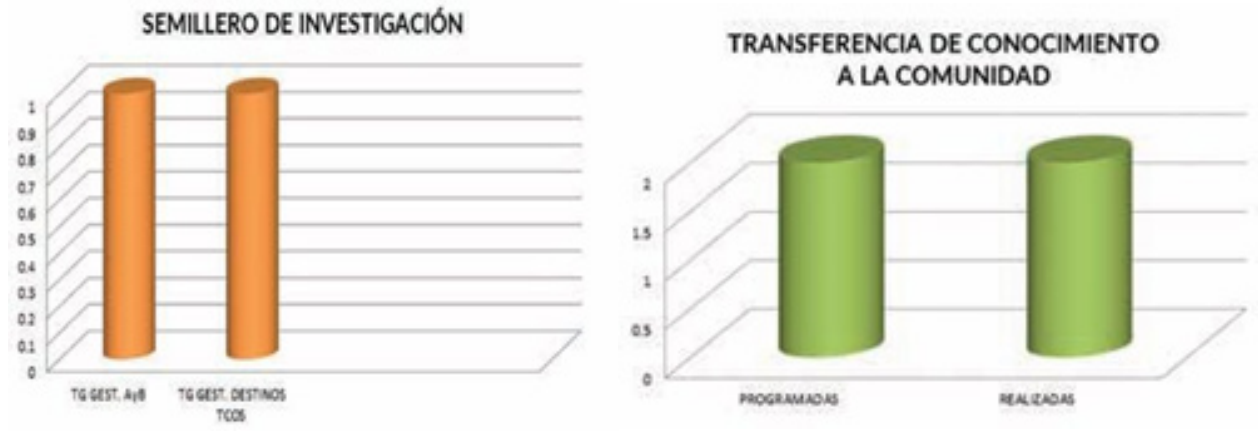

Fuente: Pérez y Carrillo, 2020.

La articulación entre lo asimilado en los ambientes de formación, y la experiencia real de la praxis inmersa sobre el territorio en el contexto de la investigación permitió la generación de conocimiento en los aprendices semilleros de investigación. El aporte se refleja e impacta directamente en el fortalecimiento en las competencias de investigación aplicada de manera vivencial, expresadas en sensibilización, reflexión y experimentación, a través del trabajo de campo en territorio real natural y la inmersión con la población campesina.

Dichas acciones estimulan el análisis del pensamiento crítico y el razonamiento de manera significativa como parte de la construcción de saberes; apoyada en el aprendizaje autónomo. Asimismo, compuesto por 
el desarrollo de habilidades y destrezas para el logro de sus competencias; gracias a la apropiación de conocimientos y el ejercicio de desempeño en la praxis real en el contexto; para la entrega del producto en el que se dio el proceso y tratamiento del proyecto de investigación.

\section{Discusión de resultados}

En los resultados obtenidos se detalla la identificación de 11 productos agrícolas tradicionales y ancestrales como son sagú, arrayanes, malanga, malangay, coyo blanco con morado, coyo amarillo con morado, manguzán, caimitos, nísperos, frijol baluy y bejuco batata, de producción exclusiva en la provincia de Oriente en Cundinamarca. Asimismo, se consiguió la creación de 4 huertas caseras, motivando e incitando activar las buenas prácticas agrícolas a través del uso de conocimientos, herramientas y técnicas tradicionales de siembra y generando alto valor cultural. Lo anterior, para contribuir en pro de mejorar la "seguridad alimentaria" por medio del fomento y desarrollo de huertas caseras, las cuales se toman como una alternativa de solución para acceder a los productos agrícolas orgánicos, endémicos y cultivados de forma tradicional.

El origen de los motivos por rescatar los productos agrícolas se dio a partir del interés en la comunidad local por recordar y reconocer la cultura que gira alrededor de los platos preparados desde la base en las muestras agrícolas resultado de la tierra. De esta manera comprendiendo y aprovechando la oportunidad existente de una gran variedad de saberes orales y tradiciones oriundos de la región, con posibilidad de recuperar y lograr que perduren en el tiempo.

Al respecto de estos resultados, se coincide con las siguientes afirmaciones. De acuerdo con la Convención para la salvaguardia del patrimonio cultural inmaterial (2003), se entiende por patrimonio cultural inmaterial:

Los usos, representaciones, expresiones, conocimientos y técnicas -junto con los instrumentos, objetos, artefactos y espacios culturales que les son inherentes- que las comunidades, los gruposy en algunos casos los individuos reconozcan como parte integrante de su patrimonio cultural. Este patrimonio cultural inmaterial, que se transmite de generación en generación, es recreado constantemente por las comunidades y grupos en función de su entorno, su interacción con la naturaleza y su historia, infundiéndoles un sentimiento de identidad y continuidad y contribuyendo así a promover el respeto de la diversidad cultural y la creatividad humana. (UNESCO, 2003, p. 4)

Por ello, se reconoce la cultura culinaria como expresión propia de una comunidad con características particulares. Es reflejada en la forma de alimentarse, los saberes de tipo tradicional empleados en la preparación y qué ingredientes son los utilizados adecuadamente para garantizar la 
conservación de esa identidad cultural alrededor del alimento. Corresponde al referente alusivo de memoria y de identidad desde el punto de vista antropológico (Matta, 2012).

De acuerdo con Molano (2007), el incentivar la práctica para rescatar las expresiones culturales de una sociedad, con el objetivo de afianzar la identidad cultural, contribuye en el tejido social local. Entonces, el resultado de las actividades participativas conocidas en este proyecto como Mingas evidencian como producto la recopilación de 40 recetas culinarias preparadas a base de los productos agrícolas rescatados. Lo anterior, con fines de candidatura para reconocimiento como patrimonio material e inmaterial (gastronómico-cultural) representativo de la región.

De esta manera, favorece la conservación de tradiciones ancestrales alrededor del núcleo familiar; fortaleciendo de manera significativa en las áreas del sector productivo, ambiental, sociocultural, tecnológico y en el centro de formación SENA. En este sentido, el soporte de valoración se encuentra en la matriz de marco lógico de seguimiento, la cual se construyó con valores cualitativos y cuantitativos, cumpliendo con la estimación socioeconómica global. Además, con la identificación y la propuesta de 3 circuitos turísticos en la zona se da la opción de viaje para personas nacionales y extranjeros con el fin de hacer reconocimiento de la región, aprovechando los recursos naturales y culturales vigentes; así como posibilitando otro ingreso y forma de vida para la comunidad.

La investigación desarrollada en la región aporta a la calidad de vida de los habitantes de la provincia de Oriente, porque permite incentivar la producción y el consumo de productos agrícolas endémicos, transformados en platos ancestrales y tradicionales rescatados. De igual manera, exalta el valor patrimonial cultural que representa para el departamento de Cundinamarca.

\section{Conclusiones}

A pesar de las dificultades del territorio y la escasez de semillas endémicas, se realizó la recuperación de 11 productos agrícolas tradicionales y ancestrales de producción exclusiva en la provincia de Oriente, en Cundinamarca. Dicha actividad fue apoyada con la participación voluntaria de la comunidad, quienes contribuyeron en la búsqueda de las semillas casi extintas a consecuencia del fenómeno de oferta-demanda y la pérdida tradicional de consumo en los hogares de la región.

Con los productos agrícolas (arracacha, guatila, coyo amarillo con morado, coyo banco con morado, malanga, chonque, calabaza, batata de bejuco, frijol balui, sagú, níspero, arrayan, manguzán y caimito de la región) se logró recopilar preparaciones tradicionales cuya técnica de elaboración es casera y aún se conservan en la memoria de familias por transmisión vía oral. 
Estas preparaciones son tortas, coladas, hervidos, sudados, sopas, caldos, asados, envueltos, arepas, almojábanas, panes, bebidas frías y calientes. A continuación, se mencionan entre otras las sopas o entradas como la sopa de bolas, sancocho de coyo y malanga. En los platos principales está el arroz orientuno y el cerdo confitado. En los dulces y confituras se encuentra la torta de calabaza y el pan de sagú (con su método de cocción horneado). Dentro de las bebidas está la colada de sagú y bebida de manguzán. En los pasabocas se ubica la torta de cidra (guatila) caramelizada y los palitos de malanga. Por último, las ensaladas las cuales corresponden a la criolla y la local. Cada una de estas recetas se encuentra documentadas a través de una cartilla didáctica de recetas, como producto de la investigación para el centro de formación SENA y los stakeholders.

Las recetas culinarias recopiladas están basadas en las semillas rescatadas. Por ello, para su puesta en práctica y conservación generacional se hizo necesario incentivar la producción por medio de 4 huertas caseras bajo parámetros de estudio que permitieran obtener un producto estándar con calidad alta; lo que requirió del uso de conocimientos, herramientas y técnicas tradicionales de siembra y valoración cultural propia de la zona. Estos referentes facilitaron un proceso de siembra tradicional planificada, apoyada en el uso de fertilizantes 100\% orgánicos y con el acompañamiento y seguimiento técnico moderno; en búsqueda de optimizar recursos a partir de una producción agrícola que fortalezca el nivel nutricional de estos bienes culinarios, cosechados en el año 2019.

En suma, este proyecto permitió contribuir a la cultura de "seguridad alimentaria" por medio del fomento y desarrollo de huertas caseras. Estas son tomadas como una alternativa de solución para acceder a productos agrícolas endémicos cultivados de forma tradicional y con concepto orgánico. Asimismo, que faciliten el acceso a una alimentación saludable y económica con el fin de ir fomentando progresivamente en la base alimentaria los productos ancestrales de la región.

El ejercicio del proyecto investigativo favoreció la interacción con la comunidad mediante coloquios y mingas realizadas en cada una de las fincas de las huertas caceras. Allí los participantes invitaban a sus vecinos, familiares y amigos, concibiendo como resultado el interés de la generación joven (aprendices del semillero, habitantes locales, instructores e investigadores del SENA) alrededor de las recetas rescatadas, los productos agrícolas endémicos, las técnicas, los métodos, los procedimientos de preparación y cocción, el manejo de tiempos para alistamiento y conservación. Además, exponiendo la importancia de las tradiciones culinarias, el valor y el respeto hacia estas y su compromiso como nuevos difusores del conocimiento ancestral adquirido.

Al comprender el potencial culinario de la región, la comunidad local toma conciencia de sus recursos y se incentiva a proponer actividades para dar a conocer su riqueza cultural alrededor del alimento ancestral y tradicional, 
reflejado en el trazado de tres circuitos turísticos. Esta propuesta queda para que las autoridades locales validen e incorporen en el inventario turístico y se gestione el respectivo acto administrativo local que permita incorporarlos en la oferta turística con su adecuada promoción.

La comunidad de la provincia Oriente recibió de forma positiva los resultados parciales compartidos sobre la investigación. En primer lugar, despertó el interés en los entes gubernamentales por apoyar el rescate y conservación de la riqueza y diversidad agrícola de los productos endémicos locales. En segundo lugar, se encontraron ventajas y oportunidades en alternativas de generación económica fomentando otros ingresos, bienestar y promoción turística para dinamizar el desarrollo de la región.

Finalmente, los aprendices pertenecientes al centro de formación SENA, en los semilleros de investigación como eje central del proceso investigativo, lograron fundamentar conceptos y llevar a las praxis los conocimientos y procedimientos adquiridos, de acuerdo con las actividades que realizaron a nivel de impacto del sector productivo, ambiental y sociocultural. Dichos jóvenes se encontraron con una realidad ligada al campo y sus quehaceres diarios, hechos que permitieron en cada uno sensibilizarse con la comunidad, tomar conciencia sobre el valor de las sociedades, sus costumbres, principios y valores; incluso generando una visión amplia del entorno y, en general, les ofreció herramientas para poder aplicarlas en su vida laboral.

Porúltimo, a modo de recomendaciones, es importante brindar sostenibilidad y sustentabilidad a los proyectos de investigación aplicada que se realizan desde los entes de formación técnica, tecnológica y profesional. Se siguiere a las autoridades locales fortalecer alianzas estratégicas de comercialización directa con el sector productivo y los stakeholders.

Se sugiere a las autoridades locales que mediante un acuerdo municipal se gestione lo pertinente para la inclusión de los resultados de esta investigación en el inventario turístico y promoción de estos. Asimismo, con fines de candidatura para reconocimiento como patrimonio material e inmaterial (gastronómicocultural) representativo de identidad local-regional. 


\section{Referencias bibliográficas}

Acosta López, M. A. (2018). Sabores locales [Trabajo de grado, Pontificia Universidad Javeriana]. https://repository.javeriana.edu.co/bitstream/ handle/10554/36097/Tesis\%20Uno.pdf?sequence=1\&isAllowed=y

Angélica. (2016, 3 de marzo). Población y muestra. Metodología. http:// m3todologia1.blogspot.com/2016/03/poblacion-y-muestra.html

Creswell, J y Poth, C. (2018). Qualitative Inquiry y Research Design: Choosing Among Five Approaches. SAGE Publications

Hernández Sampieri, R., Fernández, C. y Baptista, P. (2014). Metodología de la Investigación (6a ed.). McGrw Hill.

Matta, R. (2011). Posibilidades y límites del desarrollo en el patrimonio inmaterial. El caso de la cocina peruana. Revista Apuntes, 24(2), 196-207. http://www.scielo. org.co/scielo.php?script=sci_arttext\&pid=S1657-97632011000200006

Matta, R. (2012). El patrimonio culinario peruano ante UNESCO. Algunas reflexiones de gastro-politica (Working Paper Series). Desigualdades.net. https://www. desigualdades.net/Resources/Working_Paper/28_WP_Matta_Online.pdf

Meléndez, J. y Cañez De la Fuente, G. (2009). La cocina tradicional regional como un elemento de identidad y desarrollo local: el caso de San Pedro El Saucito, Sonora, México. Revista Estudios Sociales, 17, 183-204.

Molano, O. L. (2007). Identidad cultural un concepto que evoluciona. Revista Opera, (7), 69-84.

Monje Álvarez, C. A. (2011). Metodología de la investigación cuantitativa y cualitativa. Guía didáctica. Universidad Surcolombiana. https://www.uv.mx/ rmipe/files/2017/02/Guia-didactica-metodologia-de-la-investigacion.pdf

Ortiz, M. y Borjas, B. (2008). La Investigación Acción Participativa: aporte de Fals Borda a la educación popular. Revista Espacio Abierto, 17(4), 615-627.

Pérez, S. L. y Carrillo, L. E. (2020). Lo que no se narra se olvida: De la semilla a la mesa. Publicaciones SENA.

Rodríguez, R. J. (2003, 10 de julio). Cómo analizar cuantitativamente datos cualitativos. Gestopolis. https://www.gestiopolis.com/como-analizarcuantitativamente-datos-cualitativos/

Tesis de investigadores. (2012, 9 de enero). Población y muestra. Tesis de investigación. http://tesisdeinvestig.blogspot.com/2012/01/.

UNESCO. (2003). Convención para la salvaguardia del patrimonio cultural inmaterial 2003. http://portal.unesco.org/es/ev.php-URL_ID=17716\&URL_ DO=DO_TOPIC\&URL_SECTION=201.html 